Aldona Kubica ${ }^{1}$ Michał Kasprzak², Karolina Obońska², Ewa Laskowska², Anna Andruszkiewicz ${ }^{1}$, Przemysław Paciorek²

${ }^{1}$ Department of Health Promotion

2Department of Cardiology and Internal Medicine

Nicolaus Copernicus University, Collegium Medicum in Bydgoszcz, Poland

\title{
Impact of health education on adherence to clopidogrel and clinical effectiveness of antiplatelet treatment in patients after myocardial infarction
}

\author{
Corresponding author: \\ Aldona Kubica, MD, PhD \\ Department of Health Promotion, \\ Nicolaus Copernicus University, \\ Collegium Medicum \\ 3 Techników Street \\ 85-801 Bydgoszcz, Poland \\ Tel.: + 48660772077 \\ Fax: + 48525854024 \\ E-mail: aldona.kubica@gmail.com
}

Folia Medica Copernicana 2015: Volume 3, Number 4, 154-159 10.5603/FMC. 2015.0010

Copyright (C) 2015 Via Medica ISSN 2300-5432

\begin{abstract}
Non-adherence rates to antiplatelet drugs in patients with acute myocardial infarction (AMI) range from $13 \%$ to $60 \%$. We aimed to evaluate whether individual health education can improve adherence to treatment with clopidogrel in patients after AMI. This was a prospective, single-center, randomized clinical trial with a 12-month follow-up. Patients with AMI treated with percutaneous coronary intervention (PCl) were enrolled. The primary endpoint was defined as non-adherence to clopidogrel during follow-up (drug availability $\leq 80 \%$ ). Secondary endpoints included platelet function assessment, adverse cardiovascular (CV) events (CV death, PCI for ACS, unscheduled CV hospitalization). There were 191 patients enrolled in the study and divided into two groups: the individual education (IE) group (100 patients) and the standard treatment (ST) group (91 patients). Adherence to the treatment with clopidogrel based on the data from the National Health Fund did not differ significantly between the IE and ST groups [76.7\% (30.7-99.7\%) v. 84.4\% (46.5-99.7\%); $p=0.25]$. There was a substantial difference in the prevalence of unscheduled CV hospitalizations between both groups, IE and ST respectively [22 (22.0\%) v. 10 (11.0\%); $p=0.042$ ]. The rate of $\mathrm{CV}$ death and $\mathrm{ACS}$ treated with $\mathrm{PCl}$ during follow-up was low and did not differ between groups. In conclusion, the program of individual health education did not improve adherence to treatment with clopidogrel. The expected benefits of medication are not achievable at current levels of adherence. The self-reported adherence assessment is unreliable and cannot be used for effective treatment guidance. Key words: health education, adherence to clopidogrel, antiplatelet therapy, myocardial infarction
\end{abstract}

Folia Medica Copernicana 2015; 3 (4): 154-159

\section{Introduction}

Antiplatelet therapy is the cornerstone of the treatment in patients with acute coronary syndrome (ACS). Pharmacodynamic response to clopidogrel shows substantial interpatient variability. Patients with coronary artery disease (CAD) and lesser platelet inhibition in response to clopidogrel appear to be at increased risk for cardiovascular events [1, 2]. Low adherence to treatment is probably the major cause of clopidogrel "resistance" [3-5]. Despite the importance of secondary prevention, non-adherence rates to prescribed, evidence-based medicines for patients with acute myocardial infarction (AMI) range from $13 \%$ to $60 \%$ [6]. Moussa and Colombo [7] highlighted the necessity of patients' education regarding the risks and benefits of dual antiplatelet treatment to avoid premature discontinuation of the drug. Nevertheless, no standard approach has yet been developed.

The aim of this study was to evaluate whether individual health education can improve adherence to treatment with clopidogrel leading to more efficient inhibition of the P2Y12 receptor in patients after AMI.

\section{Methods}

Study population and recruitment

This prospective, single-center, randomized clinical trial with a 12-month follow-up was conducted at Antoni Jurasz University Hospital (Collegium Medicum, 
Nicolaus Copernicus University, Bydgoszcz, Poland). The recruitment phase of the study was from 1st October 2010 until 31st October 2011. All patients with AMI (both ST-elevation MI and non-ST elevation MI) and treated with percutaneous coronary intervention $(\mathrm{PCl})$ were screened for eligibility. The exclusion criteria were defined as follows: the need for prolonged use of heparin or fondaparinux, oral anticoagulant therapy, bleeding disorders (including thrombocytopenia $<100 \times 10^{3} / \mu \mathrm{L}$ ), anemia (HGB $<10.0 \mathrm{~g} / \mathrm{dL}$ ), active inflammation, heart failure in NYHA class III and IV, and life expectancy $<1$ year.

Two hundred patients, who gave informed written consent, were randomly assigned $1: 1$ to receive additional individual educational intervention (EI) or standard treatment (ST). Nine patients withdrew their consent during follow-up (all from the ST group), thus the study population comprised of 191 patients, for whom the follow-up data were available. The study population characteristics are shown in Table 1.

In-hospital management and discharge treatment recommendations strictly adhered to the European Society of Cardiology guidelines. Patients received a $600 \mathrm{mg}$ loading dose and a $75 \mathrm{mg}$ maintenance dose of clopidogrel in combination with aspirin doses of $300 \mathrm{mg}$ and $75 \mathrm{mg}$, respectively. To avoid additional confounding factors, concomitant therapy was standardized and included bisoprolol, perindopril and simvastatin, if no contraindications were present. Before being discharged from the hospital all patients obtained information regarding the need for systematic intake of the prescribed drugs as well as the hazards associated with its premature termination. Additionally, they were provided with a written warning, enclosed in the discharge letter, that read: "Do not stop treatment prematurely! Earlier discontinuation of the treatment may result in stent thrombosis and sudden cardiac death!". Follow-up visits were scheduled at 3, 6, and 9 months after discharge. During every visit ADP-induced platelet aggregation (ADP-PA) and vasodilator-stimulated phosphoprotein phosphorylation (VASP) were assessed. Moreover, patients were asked to declare their adherence to the prescribed clopidogrel (systematic drug intake: YES or NO). Patients assigned to the El group were scheduled for additional educational meetings with the attending physician at the end of hospitalization and at every follow-up visit. The intent of the meetings was to explain the pathophysiology and symptoms of $C A D$, to elucidate goals and potential gain of the treatment, and to highlight the risk of premature termination of antiplatelet therapy.

Patients' adherence to the medication regimen was also verified based on the data from The National Health Fund regarding prescribed drugs purchase. Adherence was defined as the proportion of drug availability (the number of purchased clopidogrel tablets) to drug requirement (the number of clopidogrel tablets needed to complete the treatment $=$ the number of follow-up days) and was arbitrarily judged as adequate when the proportion exceeded $80 \%$.

\section{The primary endpoint}

The primary endpoint was defined as non-adherence to clopidogrel during follow-up (drug availability $\leq 80 \%)$.

\section{Secondary endpoints}

Secondary endpoints included platelet function assessment (ADP-PA, VASP), adverse cardiovascular (CV) events (CV death, PCI for ACS, unscheduled CV hospitalization, combined of any adverse CV event).

The study protocol has been approved by The Ethical Committee of the Nicolaus Copernicus University.

\section{Platelet function assessment}

The ADP-PA examination was performed using impedance aggregometry. Whole blood was tested using a Multiplate Analyzer (Medical Cyclotron, Munich, Germany). The platelet aggregation rate was assessed as the area under the curve of the aggregation curve $(A \cup C)$ and was expressed in units of aggregation (U). For the determination of VASP phosphorylation in whole blood samples, a standardized reagent - Purified Mouse Anti-VASP (Becton, Dickinson \& Co., Franklin Lakes, NJ USA) and flow cytometer (Becton, Dickinson \& Co., Franklin Lakes, NJ USA) were used. VASP was determined based on fluorescence of blood samples after the addition of the monoclonal antibody. The platelet reactivity index (PRI) was defined as the mean fluorescence intensity (MFI) after incubation with PGE1 and ADP according to the following formula:

$$
\mathrm{PRI}=\left[\left(\mathrm{MFI}_{(\mathrm{PGE})}-\mathrm{MFI}_{(\mathrm{PGE} 1+A D P)} / \mathrm{MFI}_{(\mathrm{PGE} 1)]} \times 100 .\right.\right.
$$

\section{Statistical analysis}

According to the Shapiro-Wilk test, the investigated continuous variables were non-normally distributed, therefore, they were reported as medians and interquartile ranges (IR). For comparisons between two and three groups, the Mann-Whitney unpaired rank sum test and the Kruskal-Wallis one-way analysis of variance were used, respectively. Categorical variables were expressed as a number of patients presenting the given feature and a percentage of patients in the analysed group. Categorical variables were compared using the $\chi^{2}$ test with the Yates' cor- 
Table 1. Characteristics of the study population [data are presented as median (upper quartile-lower quartile) or number (percentage)]

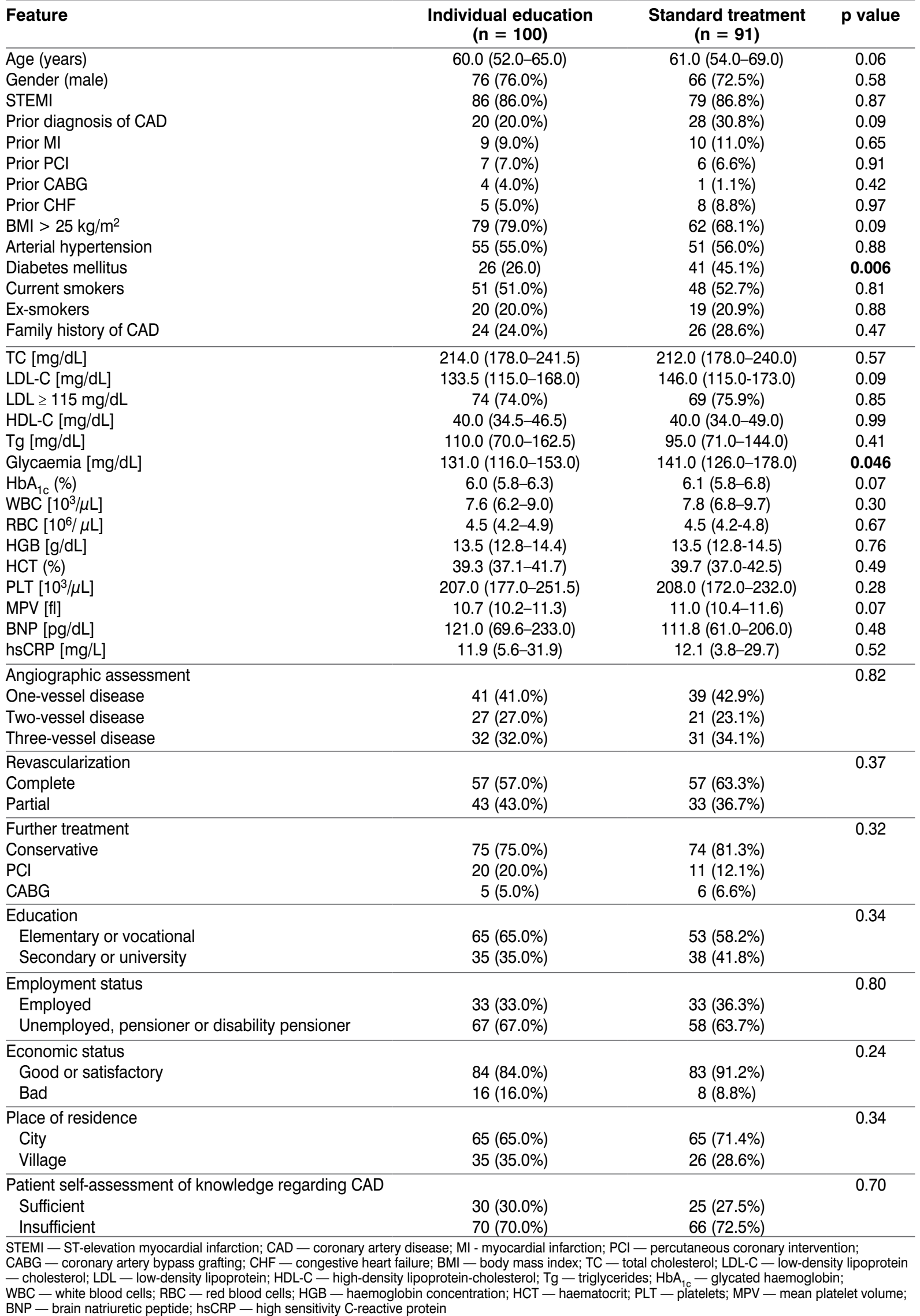


Table 2. Adherence to clopidogrel, primary and secondary endpoints [median (upper quartile-lower quartile) or number (percent)]

\begin{tabular}{|c|c|c|c|}
\hline Feature & Individual education $(n=100)$ & Standard treatment $(n=91)$ & $p$ value \\
\hline Adherence to clopidogrel & $76.7 \%(30.7-99.7 \%)$ & $84.4 \%(46.5-99.7 \%)$ & 0.25 \\
\hline Adherence $\leq 80 \%$ & $49(49.0 \%)$ & 35 (38.5\%) & 0.13 \\
\hline ADP-PA [U] & $18.0(10.0-30.0)$ & $19.5(11.0-29.0)$ & 0.56 \\
\hline PRI (\%) & $54.5(38.3-67.8)$ & $51.9(34.9-64.7)$ & 0.28 \\
\hline CV death & $0(0.0 \%)$ & $2(2.2 \%)$ & 0.44 \\
\hline PCl for ACS & $6(6.0 \%)$ & $4(4.4 \%)$ & 0.86 \\
\hline Unscheduled CV hospitalization & $22(22.0 \%)$ & $10(11.0 \%)$ & 0.042 \\
\hline $\begin{array}{l}\text { Combined of any adverse CV event } \\
\text { at follow-up }\end{array}$ & $22(22.0 \%)$ & $14(15.4 \%)$ & 0.24 \\
\hline
\end{tabular}

ADP-PA — ADP-induced platelet aggregation; PRI — platelet reactivity index; CV — cardiovascular; $\mathrm{PCl}$ - percutaneous coronary intervention; ACS - acute coronary syndrome

rection if required. The Cochran-Armitage test was used to assess the presence of a linear trend among categorical variables. Differences were considered significant at $p<0.05$. The statistical analysis was carried out using the Statistica 10.0 package (StatSoft, Tulsa, USA).

\section{Results}

Patients assigned to the El and ST groups did not differ significantly, with the exception of the prevalence of diabetes mellitus and glucose concentration on admission (Tab. 1). The evaluation of the patients' adherence to treatment with clopidogrel based on the data from The National Health Fund did not differ significantly between both groups of patients (Tab. 2). However, the prevalence of non-adherence defined as the drug availability $(\leq 80 \%)$ tended to be higher in El patients when compared with ST group (49\% v. 38.5\%; $p=0.13$ ). Interestingly, self reported proportion of adherence to treatment (declaration of systematic clopidogrel intake at each follow-up visit) was clearly distinct and amounted to $92 \%$ and $95.5 \%$, respectively. No difference between El and ST patients regarding platelet function parameters reflecting inhibition of P2Y12 receptor was found. With regard to the clinical outcome, unexpected but substantial differences in the prevalence of unscheduled CV hospitalizations appeared. The rate of CV death as well as ACS treated with $\mathrm{PCI}$ during follow-up was very low and did not differ between groups. According to multivariate analysis, female gender was the only predictor of adherence to antiplatelet treatment OR 2.504 (95\% Cl 1.254-4.999, p = 0.009).

\section{Discussion}

"Drugs don't work in patients who don't take them" - C. Everett Koop, MD. Medication adherence is an issue of growing concern for those working to improve health system performance. Promoting adherence to medications offers a rare opportunity to simultaneously improve health outcomes whilst reducing costs in patients after AMI $[8,9]$. On the basis of our previous experience [10-12], we assumed that individual, complex health education which would begin with hospitalization and continue during follow-up shall result in the improvement of patients' adherence to the treatment with clopidogrel in patients after AMI. Our health education intervention aimed to explain pathophysiology and symptoms of coronary artery disease, to elucidate goals and potential gain of the treatment and to highlight the risk of premature termination of antiplatelet therapy. However, we failed to demonstrate any benefits of this health education intervention.

Ferreira-González et al. [13] related $10.6 \%$ of non-adherence to dual antiplatelet treatment in 1,622 consecutive patients undergoing drug eluting stent (DES) implantation. However, these findings were based on patient declarations and were not verified by prescription refill control. They concluded that antiplatelet therapy discontinuation after DES implantation is not exceptional, it is usually temporary and does not appear to have a large impact on the risk [13]. Furthermore, taking into account the considerable differences between self-reported and verified data regarding prescribed drugs purchase revealed in our study, we can doubt the veracity of these results. The Myocardial Ischaemia National Audit Project Registry and the General Practice Research Database revealed that out 
of the 7543 patients after AMI only $68.6 \%$ with non-STsegment elevation myocardial infarction (NSTEMI) and $62.8 \%$ with ST-segment elevation myocardial infarction (STEMI) received a clopidogrel prescription in the first 3 months after discharge from the hospital. The adjusted odds of prescribed clopidogrel at 12 months were similar after NSTEMI 53\% (95\% Cl 51-55) and STEMI $54 \%$ (95\% Cl 52-56) [14]. This data corresponds with ours and likely reflects true adherence.

Several studies have tried to identify patients who fail to follow medical advice on discharge from hospital. This knowledge may help the health care professionals to develop quality improvement initiatives which target this high-risk population in order to improve their outcomes following AMI [15]. In our study, male gender was associated with significantly lower adherence as compared with female gender.

Melloni et al. [16] evaluated adherence to medication by means of interviewing 1,077 patients after acute coronary syndromes (ACS). At 3-month follow-up, 304 patients (28.2\%) had discontinued at least one of the prescribed medicines. Most cases of drug discontinuation (61.5\%) were self-determined. The factors independently associated with self-discontinuation included: having no or limited healthcare cover, increasing the number of medications, not using reminder tools (e.g., pillbox), lower education status, and dialysis [16]. On the basis of patient interviews Garavalia et al. [6] defined reasons for non-adherence to prescribed medication in patients after AMI. Adverse effects that were painful and interfered with daily life constituted the most common reason for discontinuing the use of medication.. Less common reasons for discontinuation were: prescription confusion, cost, distrust in medicine, healthcare system, and preference for alternative therapies. Reasons for clopidogrel discontinuation were duration confusion, adverse effects and cost [6]. According to Muntner et al. [17], the risk for low clopidogrel adherence was higher in patients who reported, prior to $\mathrm{PCl}$, taking smaller doses of medication due to cost or had difficulty filling prescriptions, had difficulty reaching their primary physician, or were uncomfortable asking their doctor for advice. Sengstock et al. [18] highlighted the need for corrective measures to commence on hospitalization and involve practitioners who can follow patients after discharge. A solid, ongoing relationship between patients, their primary-care physicians and cardiologists is essential regarding this issue [18]. Taking into account the findings from the previous studies, we continue with the evidence-based treatment initiated in the hospital [10-12, 19]. To build mutual trust, every patient was accompanied by the same physician during hospitalization and follow-up. Nevertheless, our educational intervention failed.
Ho et al. [20] tested the impact of multifaceted intervention and usual care on adherence to medication regimens in 253 patients with ACS. The intervention lasted for 1 year following discharge and comprised pharmacist-led medication, patient education, collaborative care between pharmacist and patient's primary care clinician and/or cardiologist, and 2 types of voice messaging (both educational and medication refill reminder calls). Using the same definition of primary endpoint that we used, a greater proportion of intervention patients adhered to clopidogrel, statins and ACEI/ARB but not $\beta$-blockers. However, this did not lead to any differences in the proportion of patients who achieved blood pressure and LDL-cholesterol level goals [20]. McDonald et al. [21] performed a systematic review of the published randomized controlled trials (RCTs) of interventions aimed at assisting patients' adherence to prescribed medications. Interestingly, $49 \%$ of the interventions tested (19 out of 39 in 33 studies) were associated with statistically significant increases in medication adherence and only 17 reported statistically significant improvements in the treatment outcomes. Almost all the interventions that were effective for long-term care were complex, and included combinations of more convenient care, information, counseling, reminders, self-monitoring, reinforcement, family therapy and other forms of additional supervision or attention. Even the most effective interventions had modest effects [21].

However, the current analysis has potential limitations. Firstly, this was a single-center study and the population was relatively small. Therefore, our findings are uncertain and cannot be generalized. Secondly, drug availability does not necessarily reflect its actual intake. This, however, is a methodological limitation that should be taken into account when using this particular approach for evaluation of adherence to medication.

\section{Conclusions}

In conclusion, the program of individual health education did not improve adherence to treatment with clopidogrel. The expected benefits of medications are not available at currently achievable levels of adherence. There is a need for further innovative approaches to assist patients to follow prescribed medications in order to improve health outcomes after AMI. The self-reported adherence assessment is unreliable and cannot be used for effective treatment guidance.

\section{Funding}

The work was supported by a scientific grant from the Nicolaus Copernicus University (No. 202). 


\section{Acknowledgements}

All authors declare no conflict of interest regarding the article.

\section{References}

1. Kubica A, Koziński M, Grześk G et al. Genetic determinants of platelet response to clopidogrel. J Thromb Thrombolysis 2011; 32: 459-466.

2. Kozinski M, Bielis L, Wisniewska-Szmyt $J$ et al. Diurnal variation in platelet inhibition by clopidogrel. Platelets 2011; 22: 579-587.

3. Burke JP, Sander S, Shah H et al. Impact of persistence with antiplatelet therapy on recurrent ischemic stroke and predictors of nonpersistence among ischemic stroke survivors. Curr Med Res Opin. 2010; 26: 1023-1030.

4. Campo G, Fileti L, Valgimigli M et al. Poor response to clopidogrel: current and future options for its management. J Thromb Thrombolysis 2010; 30: 319-331.

5. Kubica A, Grześk G, Sinkiewicz W et al. Compliance, concordance, adherence w przewlekłej terapii. Folia Cardiologica 2010 : 5 : 54-57.

6. Garavalia L, Garavalia B, Spertus JA et al. Exploring patients' reasons for discontinuance of heart medications. J Cardiovasc Nurs 2009; 24: 371-379.

7. Moussa ID, Colombo A. Antiplatelet therapy discontinuation following drug-eluting stent placement: dangers, reasons, and management recommendations. Catheter Cardiovasc Interv 2009; 74: 1047-1054.

8. Tuppin P, Neumann A, Danchin N et al. Evidence-based pharmacotherapy after myocardial infarction in France: adherence-associated factors and relationship with 30-month mortality and rehospitalization. Arch Cardiovasc Dis 2010; 103: 363-375.

9. Roth GA, Morden NE, Zhou W et al. Clopidogrel Use and early outcomes among older patients receiving a drug eluting coronary artery stent. Circ Cardiovasc Qual Outcomes 2012; 5: 103-112.

10. Kubica A, Pufal J, Moczulska B et al. Skuteczność edukacji zdrowotnej u osób hospitalizowanych w klinice kariologii. Psychiatria w Praktyce Ogólnolekarskiej 2005; 5: 61-68.
11. Kubica A, Magielski P, Olejarczyk E et al. Źródła wiedzy a skuteczność edukacji zdrowotnej u osób z ostrym zawałem serca. Folia Cardiol Excerpta 2009; 4: 285-290.

12. Kubica A, Kochman W, Bogdan M et al. Wpływ przebytych zabiegów angioplastyki wieńcowej oraz hospitalizacji z powodu zawału serca na poziom wiedzy i skuteczność edukacji zdrowotnej u osób z zawałem serca. Post Kardiol Interw 2009; 15: 25-30.

13. Ferreira-González I, Marsal JR, Ribera A et al. Double antiplatelet therapy after drug-eluting stent implantation: risk associated with discontinuation within the first year. J Am Coll Cardiol 2012; 60: 1333-1339.

14. Boggon R, van Staa TP, Timmis A et al. Clopidogrel discontinuation after acute coronary syndromes: frequency, predictors and associations with death and myocardial infarction - a hospital registry-primary care linked cohort (MINAP-GPRD). Eur Heart J 2011; 32: 2376-2386.

15. Zhu B, Zhao Z, McCollam P et al. Factors associated with clopidogrel use, adherence, and persistence in patients with acute coronary syndromes undergoing percutaneous coronary intervention. Curr Med Res Opin 2011; 27: 633-641.

16. Melloni C, Alexander KP, Ou FS et al. Predictors of early discontinuation of evidence-based medicine after acute coronary syndrome. Am J Cardiol 2009; 104: 175-181.

17. Muntner P, Mann DM, Woodward M et al. Predictors of low clopidogrel adherence following percutaneous coronary intervention. Am J Cardiol 2011; 108: 822-827.

18. Sengstock D, Vaitkevicius P, Salama A et al. Under-prescribing and non-adherence to medications after coronary bypass surgery in older adults: strategies to improve adherence. Drugs Aging 2012; 29: 93-103.

19. Kubica A, Bogdan M, Olejarczyk E et al. Samoocena pacjentów jako czynnik modyfikujący skuteczność edukacji zdrowotnej. Folia Cardiol Excerpta 2008; 3: 571-577.

20. Ho PM, Lambert-Kerzner A, Carey EP et al. Multifaceted intervention to improve medication adherence and secondary prevention measures after acute coronary syndrome hospital discharge: a randomized clinical trial. JAMA Intern Med. 2014; 174: 186-193.

21. McDonald HP, Garg AX, Haynes RB. Interventions to enhance patient adherence to medication prescriptions: scientific review. JAMA 2002; 288: 2868-2879 